\title{
Yeast as a model to investigate the mitochondrial role in adaptation to dietary fat and calorie surplus
}

\author{
E. Marchi · D. Cavalieri
}

Received: 8 October 2008/Accepted: 12 November 2008/Published online: 27 November 2008

(C) The Author(s) 2008. This article is published with open access at Springerlink.com

\begin{abstract}
Several research strategies are focused towards understanding the genetic basis and molecular mechanisms that regulate uptake, synthesis, deposition, and mobilization of lipids, in the context of energy homeostasis. Because of the complexity of the problem, major input comes from the use of model systems. The aim of this work was to test the feasibility of using yeast as a model organism for studies related to dietary challenges due to high fat diet and investigate the correlation between FA metabolism and oxidative metabolism. In particular, we ask to what extent the utilization of oleic acid is dependent on mitochondrial function. We studied growth on oleic acid as a sole carbon source, and oleate stress (growth in 2 and 5\% oleate) in both laboratory (BY4741 wild-type and 4 sco $1,4 s c o 2, \Delta t g l 3, \Delta t g l 4$ mutants) and natural strains, comparing the growth phenotypes with the respiratory behaviour for each strain. We confirmed that respiratory competence is fundamental for growth on oleic acid, since the respiratory deficient mutant $\Delta s c o l$ was unable to grow on oleic acid. In order to understand if the ability to use oleate as carbon source and adapt to high oleate concentrations is a general trait for the Saccharomyces cerevisiae genus, we also studied some natural strains, both diploid and haploid, identifying two meiotic derivatives of SGU90 as unable to grow in oleic acid as a sole carbon source. We investigate some aspects of mitochondrial metabolism in order to gain insights on this new finding.
\end{abstract}

Keywords Biodiversity - Fat diet - Model organism . Oleic acid · Yeast

E. Marchi · D. Cavalieri $(\bowtie)$

Department of Preclinical and Clinical Pharmacology,

UNIFI, Viale Pieraccini, 6, 50139 Florence, Italy

e-mail: duccio.cavalieri@unifi.it

\section{Introduction}

Lipid molecules are vital components of all eukaryotic cells. They are used as a concentrated energy source and as building blocks for membranes. Due to their coping with membrane properties, lipids must be maintained at nontoxic levels within the cells and, on the other hand, they must be available for their positive role. Thus lipids undergo a strongly regulated homeostasis, achieved through the interplay between several pathways including synthesis, transport, neutralization and storage. The consequences of fatty acids (FA) imbalance are dramatic, leading in mammals to many disease states, such as obesity, Type II diabetes, atherosclerosis and neurodegeneration. All these disease states are linked both to lipid homeostasis imbalance and to mitochondrial dysfunctions.

Since in western countries lipid-associated disorders are reaching epidemic dimensions, numerous research strategies are focused towards understanding the genetic basis and molecular mechanisms that regulate uptake, synthesis, deposition, and mobilization of lipids, in the context of energy homeostasis. Because of the complexity of the problem, major input comes from the use of model systems.

The aim of this work was to test the feasibility of using yeast as a model organism for studies related to dietary challenges due to high fat diet and investigate the correlation between FA metabolism and oxidative metabolism.

We build on the consideration that master regulators of fundamental mechanisms evolved at the cellular levels should be conserved throughout evolution and that several fundamental processes first elucidated in yeast showed unexpected conservation in mammalian systems [10].

The possibility to combine genetic analysis and genomewide patterns of gene expression has made yeast the best 
model for studies on fundamental cellular processes, ranging from DNA repair to aging, cell cycle, metabolism [6]. Yeast as a model for nutritional studies has several advantages: it is a facultative anaerobe organism, able to live on both fermentable and non-fermentable carbon sources. During fermentative growth, glycolysis generates all the necessary energy independently from respiratory activity, while respiration is fully activated under nonfermentative growth conditions. Yeast is able to survive without functional mitochondria (RD mutants, respiratory deficient), provided that a fermentable carbon source is made available. Strong similarities have been recognized between yeast glucose/nutrient-signalling cascade (Ras/ PKA/Msn2/4 and Sch9) with insulin and insulin-like growth factor-1 (IGF-1) signalling systems in worms, flies, mammals and humans [5]. Finally, it is possible to expose one cell type to a pure dietary challenge (eventually causing an oxidative insult) thereby controlling all the variables.

Yeast can use both saturated and unsaturated fatty acids as a carbon source and several studies have already been performed concerning yeast metabolism of oleic acid in particular: physiological characterization [12], transcriptional profiling [4], knock-out mutants screening [8].

Using yeast as a model in nutritional studies linked to high fat diet was quite a novel scientific topic, most of all because fatty acid metabolism in yeast differs from higher eukaryotes, which perform FA $\beta$-oxidation both in mitochondria and peroxisomes, while yeast uses only peroxisomes. We consider this difference an advantage rather than an obstacle, in studies aiming to gain further insights into the role of mitochondria in fatty acids metabolism and in the evolutionary reasons leading yeast to choose such a compartmentalization.

Table 1 Yeast strains

\begin{tabular}{|c|c|c|c|}
\hline Yeast strains & \multicolumn{2}{|l|}{ Genotype } & Origin \\
\hline \multicolumn{4}{|l|}{ Laboratory strains } \\
\hline BY4741 (ATCC 201388) & \multicolumn{2}{|c|}{ MATa his3 31 leu $2 \Delta 0$ met $15 \Delta 0$ ura3 $\Delta 0$} & YGSC [1] \\
\hline BY4741 $\Delta s c o 1$ & \multicolumn{2}{|c|}{ MATa his $3 \Delta 1$ leu $2 \Delta 0$ met $15 \Delta 0$ ura3 $\Delta 0$ scol::kanMX4 } & YGSC \\
\hline BY4741 $4 s c o 2$ & \multicolumn{2}{|c|}{ MATa his $3 \Delta 1$ leu $2 \Delta 0$ met $15 \Delta 0$ ura3 $\Delta 0$ sco $2:: k a n M X 4$} & YGSC \\
\hline BY4741 $\Delta \operatorname{tgl3}$ & \multicolumn{2}{|c|}{ 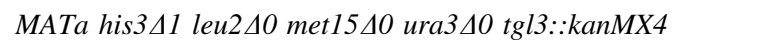 } & YGSC \\
\hline 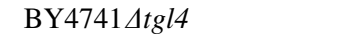 & \multicolumn{2}{|c|}{ 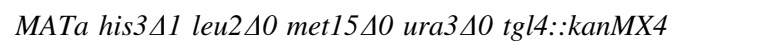 } & YGSC \\
\hline BY4741 POT1-GFP & \multicolumn{2}{|c|}{ 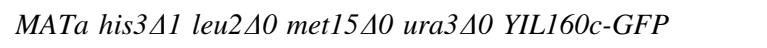 } & YGSC \\
\hline \multicolumn{4}{|l|}{ Natural strains } \\
\hline M12 & Prototrophic & San Giovese Montalcino Grape & UniFi-Cavalieri collection \\
\hline M28 & Prototrophic & San Giovese Montalcino Grape & UniFi-Cavalieri collection \\
\hline M57 & Prototrophic & San Giovese Montalcino Grape & UniFi-Cavalieri collection \\
\hline EM93 & Prototrophic & Rotten Figs (California) & UniFi-Cavalieri collection \\
\hline SG60 & Prototrophic & San Giovese industrial fermetation & UniFi-Cavalieri collection \\
\hline SGU89 & Prototrophic & San Giovese Chianti Grape & UniFi-Cavalieri collection \\
\hline SGU90 & Prototrophic & San Giovese Chianti Grape & UniFi-Cavalieri collection \\
\hline SGU114 & Prototrophic & San Giovese Chianti Grape & UniFi-Cavalieri collection \\
\hline SGU406 & Prototrophic & San Giovese Chianti Grape & UniFi-Cavalieri collection \\
\hline SGU407 & Prototrophic & San Giovese Chianti Grape & UniFi-Cavalieri collection \\
\hline SGU421 & Prototrophic & San Giovese Chianti Grape & UniFi-Cavalieri collection \\
\hline 1014 & Prototrophic & Industrial strain & $\mathrm{DBVPG}^{\mathrm{a}}$ collection \\
\hline M12 (a) & MATа ura $^{-} h$ & & UniFi-Cavalieri collection \\
\hline M28 (a) & MATa ura $^{-} h$ & & UniFi-Cavalieri collection \\
\hline M57 (a) & MATa ura $h$ & & UniFi-Cavalieri collection \\
\hline SGU90 (a) & MATа иra $h$ & & UniFi-Cavalieri collection \\
\hline $\operatorname{M12}(\alpha)$ & MATa ura $^{-} h$ & & UniFi-Cavalieri collection \\
\hline $\operatorname{M} 28(\alpha)$ & MATa ura- $h$ & & UniFi-Cavalieri collection \\
\hline $\operatorname{M57}(\alpha)$ & MATa ura $h$ & & UniFi-Cavalieri collection \\
\hline SGU90 $(\alpha)$ & MATa ura $^{-} h$ & & UniFi-Cavalieri collection \\
\hline
\end{tabular}

${ }^{a}$ Department of Vegetal Biology, Perugia (PG) 


\section{Methods}

Yeast strains and growth media

The yeast strains used in this work were both laboratory and natural strains. They are listed in Table 1. YP medium contained 1\% Yeast Extract (OXOID) and 2\% Bactopeptone (BD). Various carbon sources were added at the indicated concentrations. Oleic acid (SIGMA) was sterilized by filtration and added at the indicated concentration using as emulsifier Tween 80 at $0.2 \%$ final concentration $(\mathrm{v} / \mathrm{v})$. Solid media were obtained by the addition of $2 \%$ Agar (SIGMA).

\section{Growth tests}

For spot test analysis, yeast strains were inoculated in YP liquid medium added with $2 \%$ glucose and were grown over-night at $28^{\circ} \mathrm{C}$ in shaking. The following day, we counted with Bürker chamber the concentration of each culture and performed serial dilutions in order to reach $1 \times 10^{7}, 1 \times 10^{6}, 1 \times 10^{5}, 1 \times 10^{4} \mathrm{cell} / \mathrm{ml}$. From each one of these dilutions we spotted $10 \mu \mathrm{l}$ drop on plates containing the different growth media: YP added with $2 \%$ agar and the different carbon sources at the indicated concentration. We serially plated $1 \times 10^{5}, 1 \times 10^{4}$, $1 \times 10^{3}, 1 \times 10^{2}$ cells per spot.

\section{Fluorescence microscopy}

After $24 \mathrm{~h}$ of metabolic shift from YPD to YP added with $5 \%$ oleic acid, cultured cells were resuspended at $1 \times 10^{6}$ cells $/ \mathrm{ml}$ in $10 \mathrm{mM}$ HEPES buffer, $\mathrm{pH} 7.4$, containing $5 \%$ glucose. Rhodamine B hexyl ester (Molecular Probes) was added to a final concentration of $100 \mathrm{nM}$. After 15-30 min of incubation, mitochondrial membrane potential was visualized by fluorescence microscopy (eccitation $\lambda$ at $555 \mathrm{~nm}$, green/emission $\lambda$ at $579 \mathrm{~nm}$, red). Another equal aliquot of cells was treated with Dihydrorodamine123 (Molecular Probes) to analyze endogenous ROS production (excitation $\lambda$ at $505 \mathrm{~nm}$, blue/emission $\lambda$ at $534 \mathrm{~nm}$, green) following manufacturer instructions. Each aliquot was treated with Calcolfluor White (M2R) (Blue) to evidence cell wall in order to count total cells.

\section{Results}

Mitochondrial activity is essential for grow on oleic acid

In order to assess dependence of oleic acid on mitochondrial metabolism, we choose to analyze the growth phenotype of some previously identified mutants of Saccharomyces cerevisiae in the presence of different concentrations of oleic acid as a sole carbon source. In particular, we performed spot tests in the presence of oleic acid $0.1 \%$ (fat diet) and 2\% (high fat diet). The chosen strains were knock-out mutants of BY4741; the following genes were deleted: $\mathrm{SCOI}$, encoding a protein of the mitochondrial inner membrane necessary for the delivery of copper ions to cytochrome c oxidase and for the correct assembly of the complex; SCO2, encoding a protein anchored to the mitochondrial inner membrane, interacting with Cox $2 p$ and similar to Sco1p, which may have a redundant function with Scolp in the delivery of copper to cytochrome c oxidase; TGL3, encoding one of the triacylglycerol lipases of $S$. cerevisiae; TGLA, encoding another triacylglycerol lipase involved in triacylglycerol mobilization and degradation. Deletion of tgl3/tgl4 is associated to the obese-like phenotype of S. cerevisiae [7].

As shown in Fig. 1, all mutants except $\Delta$ scol are able to use oleic acid as a sole carbon source. The unique characteristic of 4 scol mutant is respiratory deficiency, which means that it cannot grow on any carbon source requiring respiratory mitochondrial activity.

Using a yeast strain bearing the fusion product GFP-POT1 (3-ketoacyl-CoA thiolase, peroxisomal localization) we were able to follow peroxisomal proliferation after metabolic shift of a wild-type laboratory strain from
Fig. 1 Spot test analysis of growth phenotype on standard $(0.1 \%)$ and high $(2 \%)$ oleic acid for the laboratory strain BY4741 and the four mutants Asco1, 4 sco2, $\Delta$ tgl3, $\Delta t g l 4$. Only the respiratory deficient $\Delta$ scol mutant is unable to grow on oleic acid

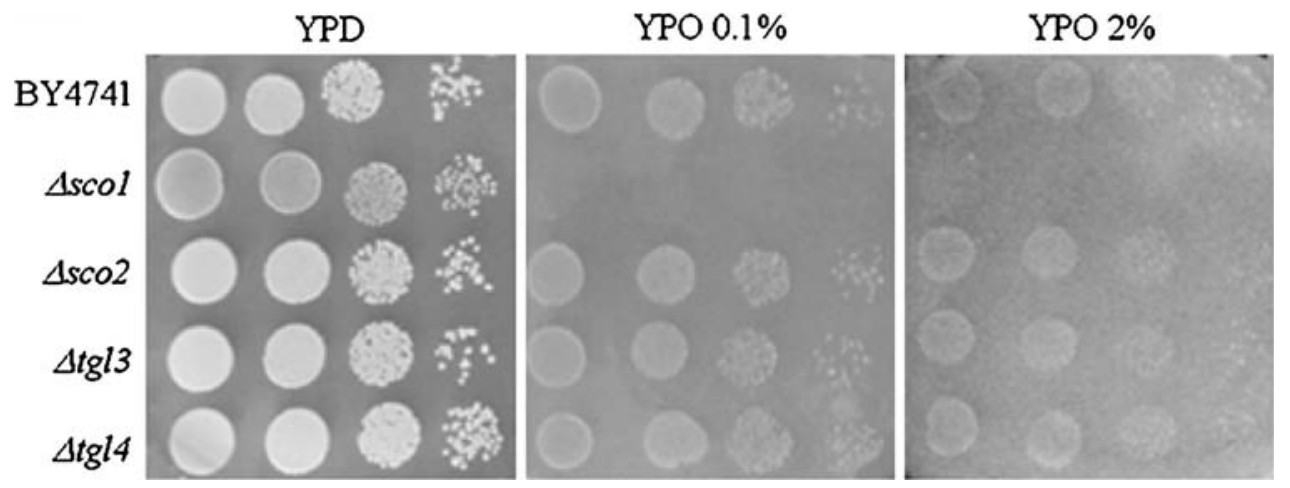


Fig. 2 Effects of metabolic shift from $2 \%$ glucose to standard $(0.1 \%)$ and high $(5 \%)$ oleic acid in liquid medium. a Peroxisomal proliferation was followed using a BY4741 strain bearing a fusion product GFP-POT1. b Mitochondrial membrane potential was detected by Rhodamine B hexyl ester staining
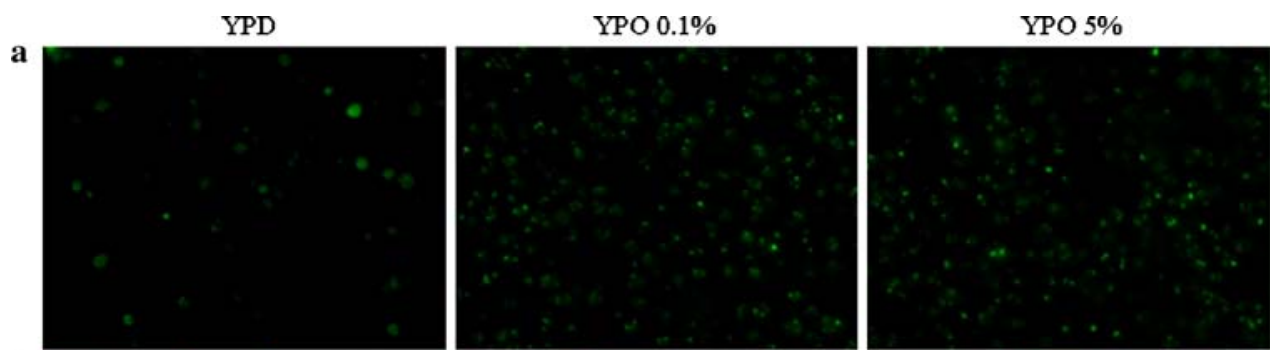

b
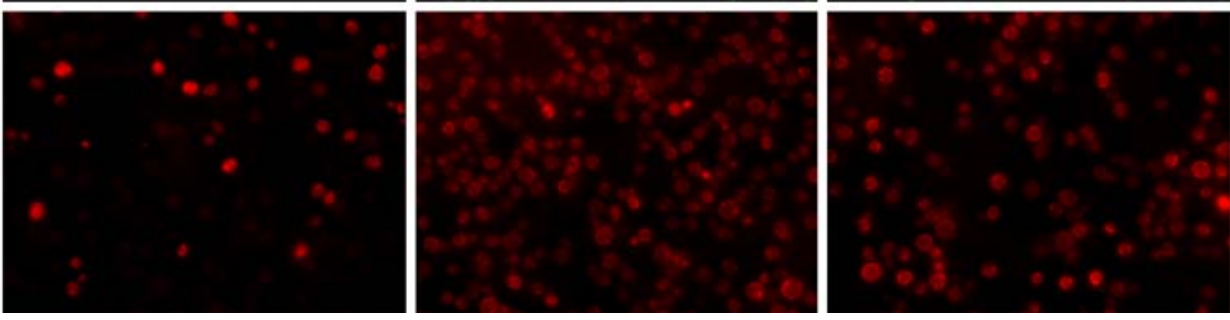

Fig. 3 Spot test analysis of growth phenotype on standard $(0.1 \%)$ and high $(2 \%)$ oleic acid compared with growth on $2 \%$ glucose for some selected diploid prototrophic natural strains

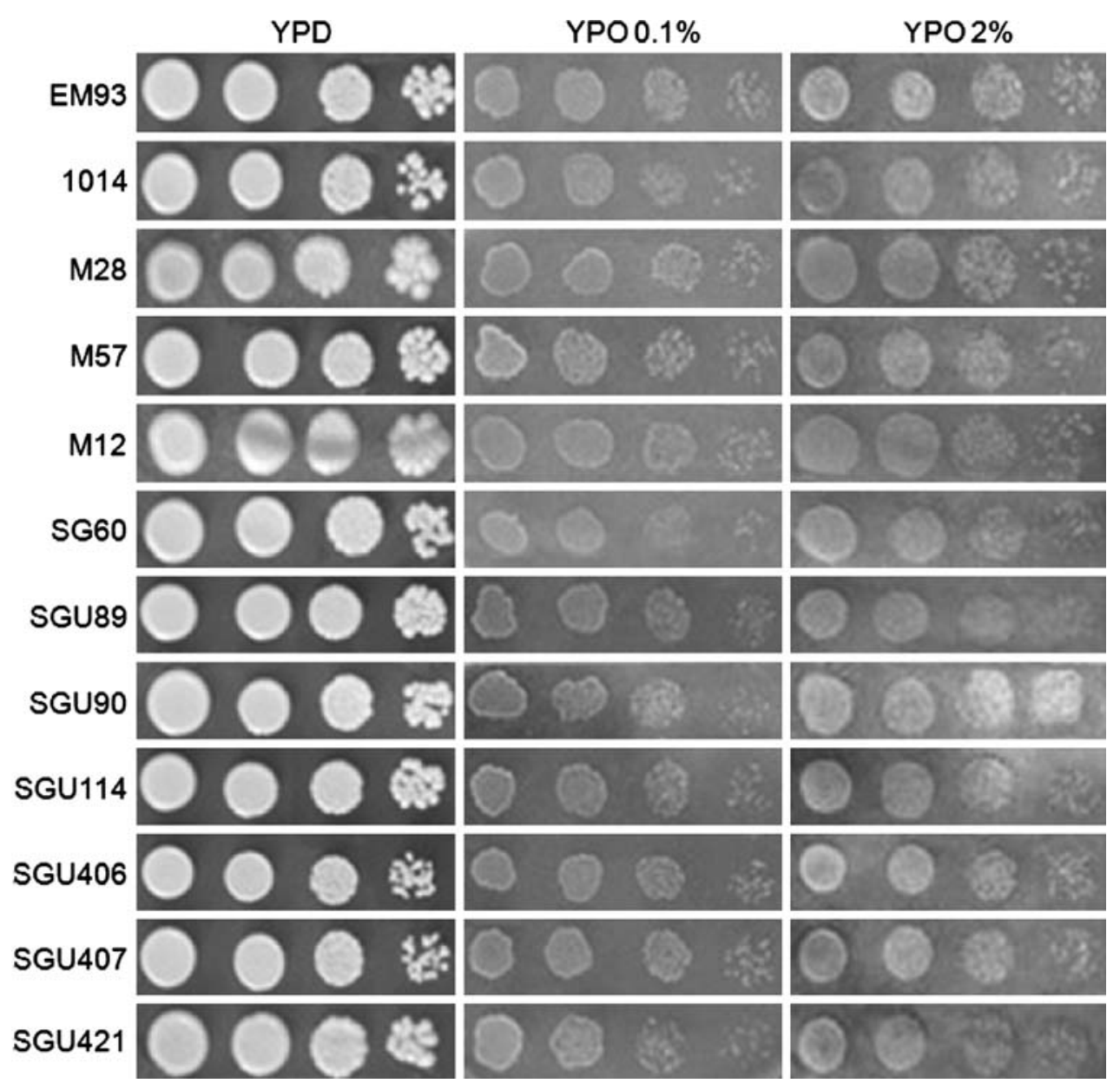

YPD to YPO 0.1 and $5 \%$. In the presence of oleic acid at both concentrations we observe intense proliferation of peroxisomes (Fig. 2a). On the same strain we performed staining with Rhodamine B hexyl-ester, a cell-permeant dye used for detecting changes in membrane potentials caused by respiratory activity, ion-channel permeability and drug binding [11]. We observed that metabolic shift from $2 \%$ glucose to oleic acid, both at 0.1 and $5 \%$ concentrations, is associated with strong activation of mitochondrial membrane potential (Fig. 2b). 
Fig. 4 Spot test analysis of growth phenotype on standard $(0.1 \%)$ and high $(2 \%)$ oleic acid compared with growth on $2 \%$ glucose for the diploid natural strains M12, M28, M57 and SGU90 and the corresponding sporulation derived heterothallic $M A T a$ and $M A T \alpha$ strains

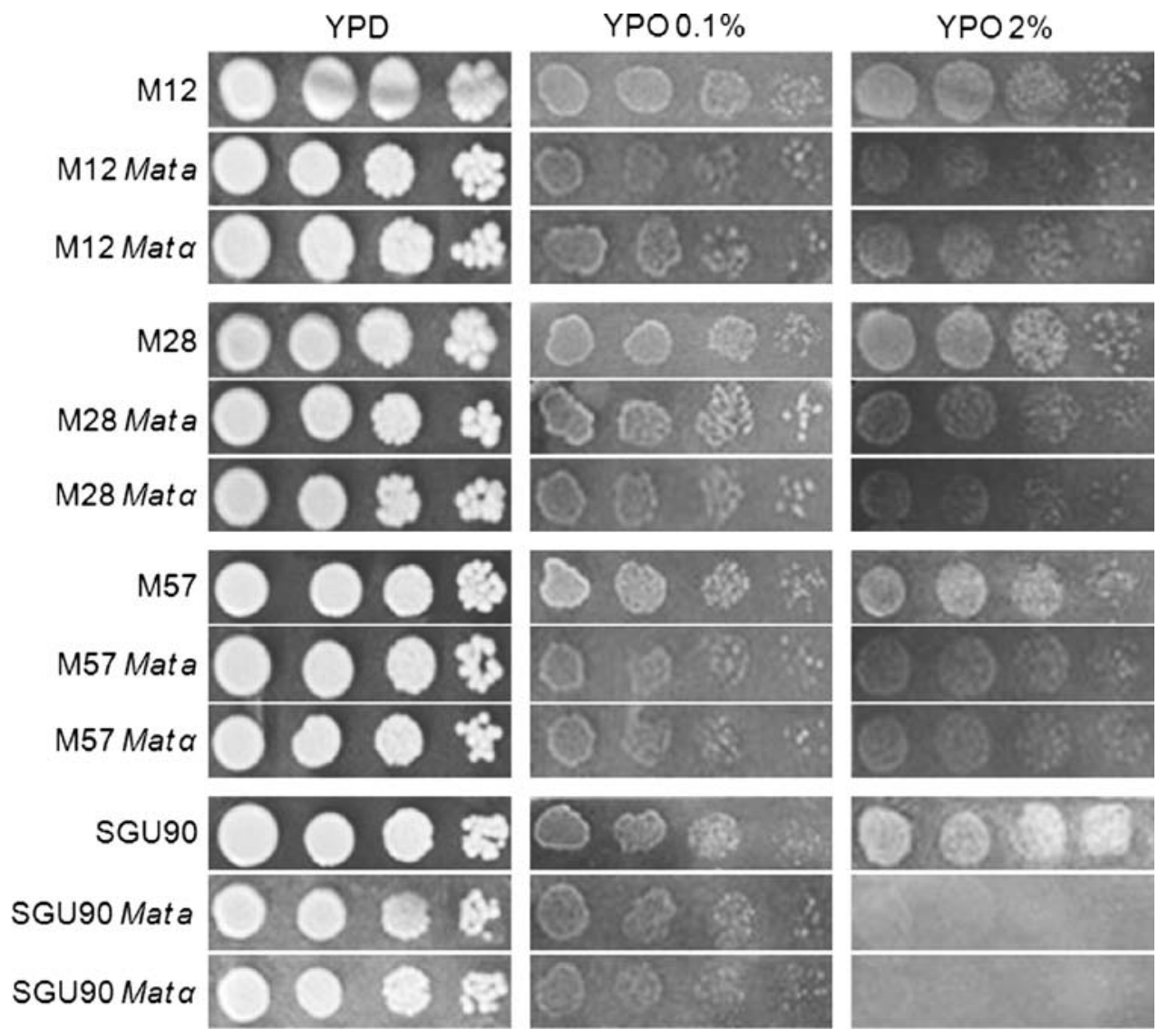

From laboratory to evolving natural populations

A limit in exporting concepts studied in yeast to mammals is represented by the fact that the standard laboratory strain is much more prone to fermentation than to respiration.

Due to this metabolic difference between natural and laboratory strains, we decided to study growth competence of a reference collection representing the Biodiversity of natural strains of $S$. cerevisiae in presence of oleic acid as a sole carbon source. As shown in Fig. 3, all the selected strains present the same ability to grow on both standard and high oleic acid.

Activation of mitochondrial metabolism was observed in all the strains studied. Oleate apparently did not cause significant increase in ROS production, except for strain SGU89 (Fig. 6b).

In order to investigate whether the strains were carrying recessive mutations relevant to oleic acid utilization we finally compared the growth phenotype on oleic acid of some natural strains with that of haploid strains derived from their sporulation, bearing a deletion of the $\mathrm{HO}$ gene, and uracil auxotrophy.

Diploid strains M12, M28 and M57 and their haploid derivatives MATa and MAT $\alpha$ strains were characterized by the same growth phenotype on both standard and high oleic acid. On the contrary, SGU90 and two haploid meiotic segregants showed a different behaviour: all three strains were able to grow on YPD and standard oleic acid, but the two haploid strain showed impaired growth on high oleic acid (Fig. 4).

In order to gain further insight into this different growth competence, we investigated mitochondrial activation and ROS accumulation in the strains of the reference collection. In Fig. 5 we show the behaviour of the reference diploid natural strain 1014, SGU89, the diploid strain SGU90, the two corresponding haploid strains MATa and $M A T \alpha$, and the reference haploid laboratory strain BY4741.

Concerning mitochondrial activation, we observed that, after staining with Rhodamine B, all strains showed comparable numbers of cells presenting activated mitochondrial membrane potential, indicating fully respiring cells (15-30\%). After metabolic shift to oleic acid as a sole carbon source, we observed an increase of mitochondrial activation: the SGU90 and SGU90 MAT $\alpha$ strains showed the relative less intense membrane potential activation (nearly 50\% increased), while the 1014 strain was the most reactive $(70 \%)$.

We also studied ROS accumulation through Rhodamine 123 staining. When exponentially growing on glucose, all 
Fig. 5 Effects of metabolic shift from $2 \%$ glucose to high $(5 \%)$ oleic acid in liquid medium. The compared strains are the standard laboratory strain BY4741, the reference natural diploid strain 1014, the two diploid "Chianti" strains SGU89 and SGU90 and the two heterothallic sporulation derived strains SGU90 MATa and MAT $\alpha$.A: Mitochondrial membrane potential was detected by Rhodamine B hexyl-esther staining. b ROS accumulation was followed by Dihydro-rhodamine 123 staining

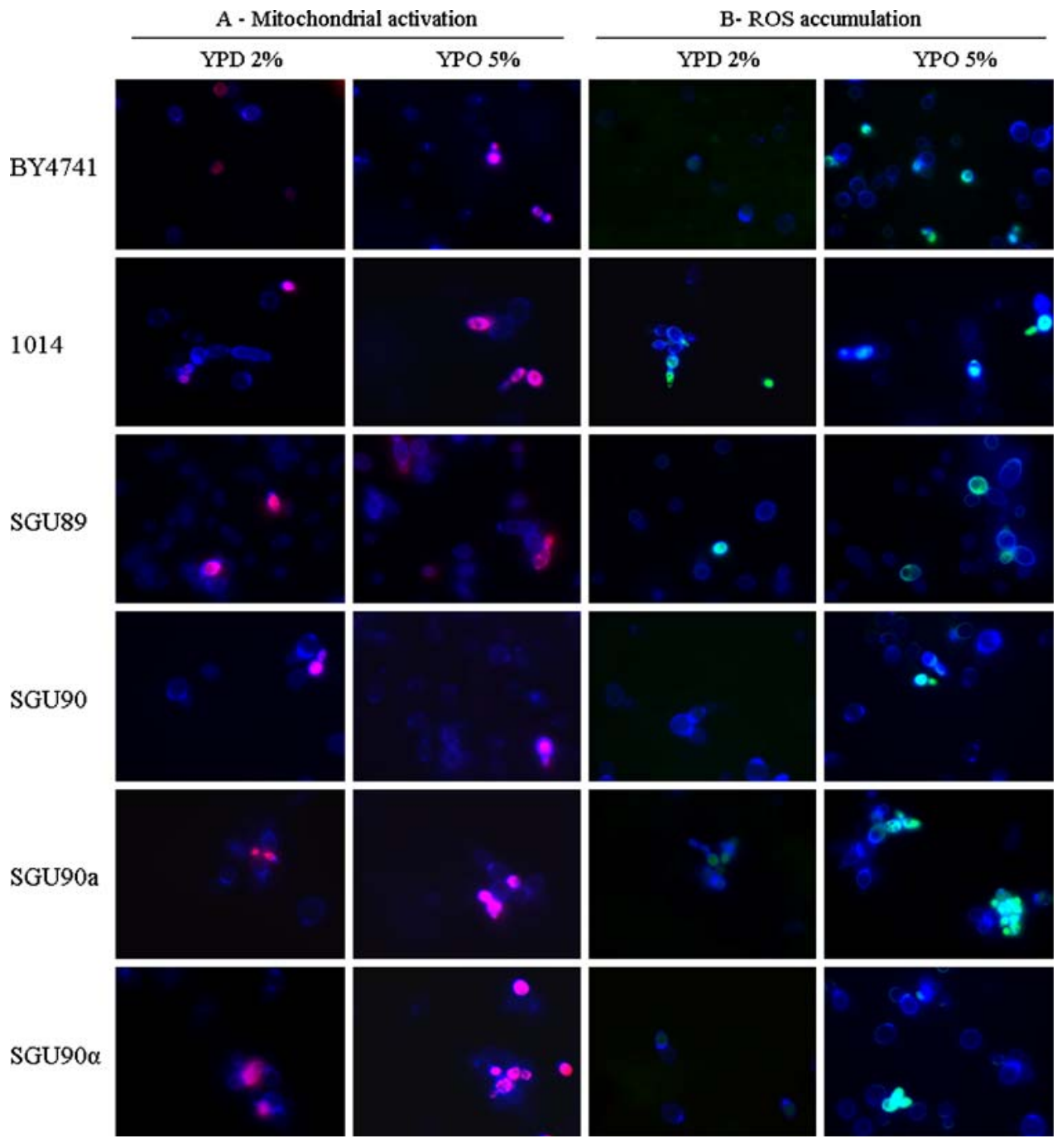

strains showed low ROS levels. After metabolic shift to oleic acid, all strains showed some ROS accumulation, in particular both haploid strains SGU90 MATa and MAT $\alpha$ and the diploid strain SGU89 showed statistically significant increase $(P<0.01)$. Due to this difference, we suggest that ROS accumulation could partly explain the impaired grow on oleic acid of the two haploid strains in the genetic background SGU90, even if the same stress factor seems not to have any negative influence on the fitness of the diploid strain SGU89 in the presence of oleic acid.

\section{Discussion}

The aim of our work is to investigate the possibility to use yeast as a model organism in Nutritional Systems Biology studies.

We present the use of a selected set of $S$. cerevisiae strains to investigate the genetic bases of the cellular and molecular response to dietary challenges, including a high fat diet.

Our approach has led to the identification of the SCOI gene as essential for growth on oleic acid as a sole carbon source. The $S$. cerevisiae $\Delta$ scol mutant bears a deletion of a gene fundamental for the correct assembly of mitochondrial cytochrome c oxidase, and so it is respiratory deficient and unable to grow on oleate. The previous study of Lockhson et al. did not identify the SCOl gene as essential for growth on oleic acid [8].

This result alone indicates that proper assembly of cytochrome c oxidase is required for growth on oleate, as well as on other non-fermentable carbon sources.

Taking into consideration the importance of respiratory competence for fatty acids metabolism and the fact that laboratory strains are more prone to fermentation rather than respiration, we decided to extend our investigation to some natural strains recovered from grapes and wine fermentations. We observed that all the selected wine strains 

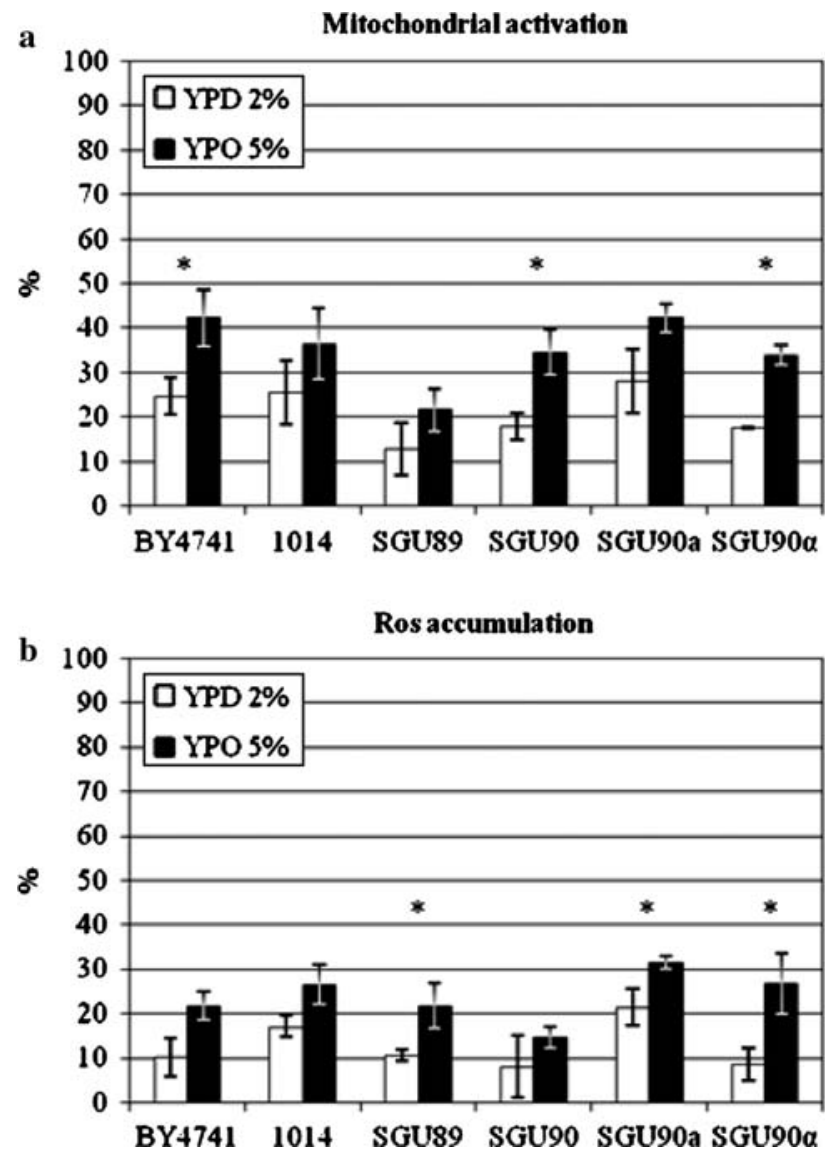

Fig. 6 Quantitative evaluation of the effects of a metabolic shift from $2 \%$ glucose to high $(5 \%)$ oleic acid in liquid medium. The percentage values were calculated as the number of stained cells over total cells in the optical field. a Mitochondrial membrane potential. b ROS accumulation. $* P<0.01$

were able to grow on both standard and high oleic acid. Nevertheless, it was noteworthy that one of the strains (SGU89) was showing significant more ROS accumulation than the other strains and a consequent reduction in mitochondrial function.

When analyzing the growth phenotype of sporulation derived heterothallic strains, we observed that the two $M A T a$ and MAT $\alpha$ strains derived from SGU90 were unable to grow in high oleic acid. In the two homothallic strains SGU90 MATa and SGU90 MAT $\alpha$ the homothallism gene $H O$ has been disrupted with a deletion cassette bearing, respectively, hygromycin and G418 resistance selective markers, and also the heterothallic strains generated in M28, M12 and M57 backgrounds underwent the same manipulation. We cannot attribute such differences in growth phenotype to the gene disruption procedure. Our observations rather suggest that the diploid homothallic strain SGU90 is heterozygous for a mutation leading to impaired fatty acid metabolism. Previous studies on natural yeast revealed that these strains show high heterozygosis for a variable number of loci $[2,3,9]$. The observation that analysis of the variability of a small set of strains uncovers interesting phenotypes linking FA metabolism and ROS accumulation underlines the importance of studying existing biodiversity and evolution in a nutritional context. Investigating the genetic features that make the two heterothallic strains different form the parental strain will indicate targets for further studies on oleic acid metabolism.

Overall our results indicate the potential of yeast in Nutritional Systems Biology. Further investigation of the collection of laboratory strain mutants and of the biodiversity of yeasts isolated from natural environments, holds great potentials for the elucidation of the fundamental mechanisms behind functional compartmentalization of FA metabolism.

Acknowledgments This work was financially supported by the Network of Excellence in Nutrigenomics, NuGO. We thank Dr. Tania Gamberi and Prof. Alessandra Modesti for useful discussions on the sco mutants. We thank Prof. Tiziana Lodi and Prof. Paola Goffrini from the Department of Genetics, Biology of Microorganisms, Anthropology, Evolution in Parma University, for useful discussion.

Conflict of interest statement None declared.

Open Access This article is distributed under the terms of the Creative Commons Attribution Noncommercial License which permits any noncommercial use, distribution, and reproduction in any medium, provided the original author(s) and source are credited.

\section{References}

1. Brachmann CB, Davies A, Cost GJ, Caputo E, Li J, Hieter P, Boeke JD (1998) Designer deletion strains derived from Saccharomyces cerevisiae S288C: a useful set of strains and plasmids for PCRmediated gene disruption and other applications. Yeast 14:115-132

2. Cavalieri D, Barberio C, Casalone E, Pinzauti F, Sebastiani F, Mortimer RK, Polsinelli M (1998) Genetic and molecular diversity in $S$. cerevisiae natural populations. Food Technol Biotechnol 36:45-50

3. Cavalieri D, Townsend JP, Hartl DL (2000) Manifold anomalies in gene expression in a vineyard isolate of Saccharomyces cerevisiae revealed by DNA microarray analysis. Proc Natl Acad Sci USA 97:12369-12374

4. Gurvitz A, Rottensteiner H (2006) The biochemistry of oleate induction: transcriptional upregulation and peroxisome proliferation. Biochim Biophys Acta 1763:1392-1402

5. Katic M, Khan CR (2005) The role of insulin and IGF-1 signaling in longevity. Cell Mol Life Sci 62:320-343

6. Kruglyak L (2008) The road to genome-wide association studies. Nat Rev Genet 9:314-318

7. Kurat CF, Natter K, Petschnigg J, Wolinski H, Scheuringer K, Scholz H, Zimmermann R, Leber R, Zechner R, Kohlwein SD (2006) Obese yeast: triglyceride lipolysis is functionally conserved from mammals to yeast. J Biol Chem 281:491-500

8. Lockshon D, Surface LE, Kerr EO, Kaeberlein M, Kennedy BK (2007) The sensitivity of yeast mutants to oleic acid implicates 
the peroxisome and other processes in membrane function. Genetics 175:77-91

9. Mortimer RK (2000) Evolution and variation of the yeast (Saccharomyces) genome. Genome Res 10:403-409

10. Resnick MA, Cox BS (2000) Yeast as an honorary mammal. Mut Res 451:1-11

11. Reungpatthanaphong P, Dechsupa S, Meesungnoen J, Loetchutinat C, Mankhetkorn S (2003) Rhodamine B as a mitochondrial probe for measurement and monitoring of mitochondrial membrane potential in drug-sensitive and -resistant cells. J Biochem Biophys Meth 57:1-16

12. van Roermund CWT, Waterham HR, Ijlsta L, Wanders RJA (2003) Fatty acid metabolism in Saccharomyces cerevisiae. Cell Mol Life Sci 60:1838-1851 